\title{
Field-Theoretic Simulations of Multi-Cylinder Configurations in VIA Lithography
}

\author{
Nabil Laachi ${ }^{1}$, Tatsuhiro I wama ${ }^{1,2}$, K ris T. Delaney $^{1}$, David Shykind ${ }^{3}$, R obert Bristol ${ }^{3}$, \\ Corey J. Weinheimer ${ }^{3}$ and G lenn H. F redrickson ${ }^{1,}$ \\ ${ }^{1}$ M aterials Research Laboratory, U niversity of California, Santa Barbara, CA 93106 \\ ${ }^{2}$ Asahi K asei E-Materials Co., Shizuoka, Japan \\ ${ }^{3}$ Intel Corporation, Hillsboro, OR 97124
}

\section{K eywords: Self-C onsistent F ield Theory (SCFT), directed self-assembly, VIA lithography, contact holes, contact multiplication, extended templates.}

\section{Introduction}

The development of new patterning tools is essential to progress in nanofabrication. The directed self-assembly (DSA) of block copolymers in particular has been shown in numerous experimental and numerical studies to provide a robust strategy for creating a variety of useful line and space patterns with low defectivity ${ }^{1-7}$.

In the context of VIA lithography, DSA of PS-PMMA in cylindrical confinement has been successfully implemented to produce PM M A VIA cylinders with a reduced critical dimension (CD) relative to a guiding prepattern ${ }^{8-11}$. Beyond hole shrink, interest in DSA also lies in contact multiplication by producing several cylinders at desired locations within a single guiding templates. A n example of such a situation and the subject of this study, includes elongated templates where single rows of multiple cylinders can self-assemble and produce VIAs with reduced CD and pitch ${ }^{12-13}$.

We use three-dimensional self-consistent field theory (SCFT) simulations to investigate the detailed structure of the microdomain ordering and the factors leading to cylindrical morphologies in contact holes ${ }^{14-15}$. The AB diblock copolymers are composed of $\mathrm{N}$ monomers, a fraction $\mathrm{f}$ of which are of type $A$ and the segregation strength is $\mathrm{CN}$. In this study, we set $\mathrm{CN}=25$ and $f=0.3$. Experimentally, the A block will denote the PM M A block while the B block will refer to PS. In the present context of VIA lithography, we used the masking technique to define the confining prepattern in a larger rectangular cell ${ }^{15}$. In addition to enforcing a vanishingly small polymer density in the wall region, the implemented mask enables various selectivity conditions at the sidewalls and top and bottom surfaces of the prepattern, modeled herein using a Flory-like parameter, $C_{w}$. For the present work we assumed a neutral top of the film, and strongly A (PMMA)-attractive sidewalls and substrate corresponding to $C_{w} N=-30$. The film height (template depth) in all cases was fixed at $100 \mathrm{~nm}$.

\section{Results and discussion}

2.1. Windows of existence and defectivity Using random and pre-established seed configurations, our SCFT simulations produce both perfect rows of VIA cylinders as well as a variety of defective morphologies. Depending on the template dimensions, rows with two (TwoCyl) or three (ThreeCyl) cylinders are formed. The most common defective morphology we observed in all our simulations consists of thin sheets of PM MA with one or two PS holes perforating the PMMA sheet. Defects with one PS hole arise in template lengths commensurate with TwoCyl morphologies while defects with two PS holes are mostly present in templates with a length commensurate with ThreeCyl morphologies. The two defects of interest are illustrated in Fig. 1.

The defect formation energies, i.e. the free energy of the defective morphology minus that of the perfect reference morphology, are plotted in Fig. 1 as a function of template length in a template of fixed width $=53 \mathrm{~nm}$. For both defects, the formation energy is a non-monotonic function of template length, with a maximum at an optimal 


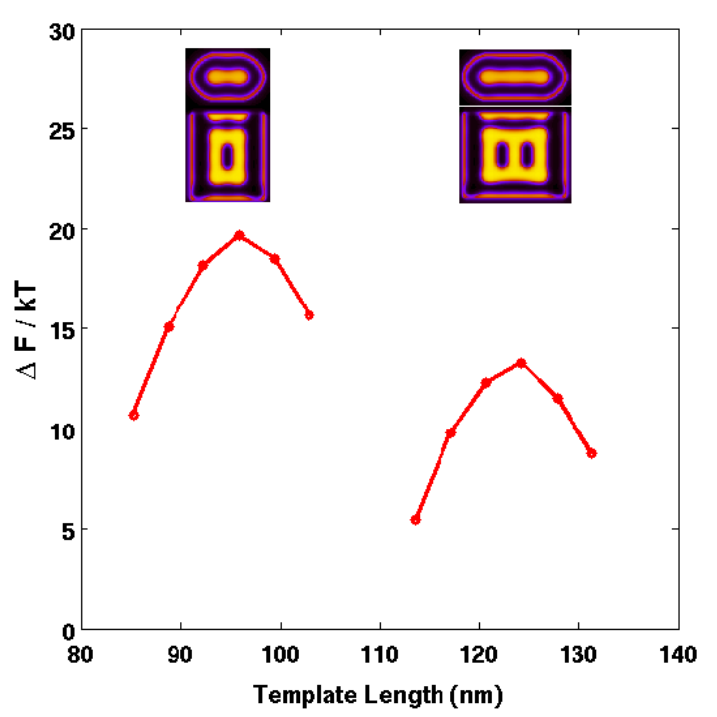

Figure 1: Plot of the formation energy for the two illustrated defects in a template of varying length and a fixed width $=53 \mathrm{~nm}$ and height $=100 \mathrm{~nm}$. Here, the sidewalls and the substrate are PMMA-attractive, $\mathrm{GN}$ $=25$ and $f=0.3$.

length. The ThreeCyl existence domain leads to lower defect energies than the TwoCyl domain, indicating a greater propensity for defect formation as the template length is increased. A Iso, for both cases, the defect formation energies are rather low, below $20 \mathrm{kT}$. Since a maximum density of 2 defects per billion requires a minimum defect formation energy of $20 \mathrm{kT}$, our simulations suggest high defectivity levels and highlight the necessity for design strategies to render defects more costly and guarantee the viability of DSA for contact multiplication.

\subsection{VIA CD}

Plots of the resulting VIA CD as a function of the length of the template for various template widths are summarized in Fig. 2. For a given length and width of the template, VIA cylinders of a TwoCyl and ThreeCyl configuration have approximately the same $C D$, the difference being less than $1 \mathrm{~nm}$. Also, the plots in Fig. 2 show that, in their respective windows of existence, the $C D$ of VIA s in TwoCyl and ThreeCyl configurations is an increasing function of both template length and width. A closer look indicates that the VIA CD growth with respect to the length of the template is linear and is independent of the width of the template. U sing linear fits to VIA CD behavior, we

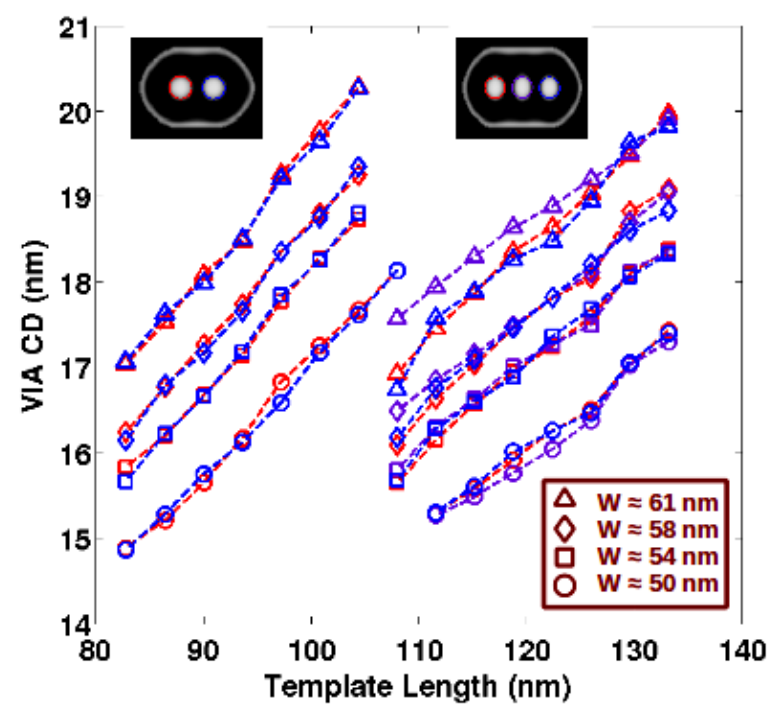

Figure 2: Plots of the CD of VIAs in TwoCyl and ThreeCyl configurations as a function of template length for various template widths, w. Each line color corresponds to the VIA indicated of the same color.

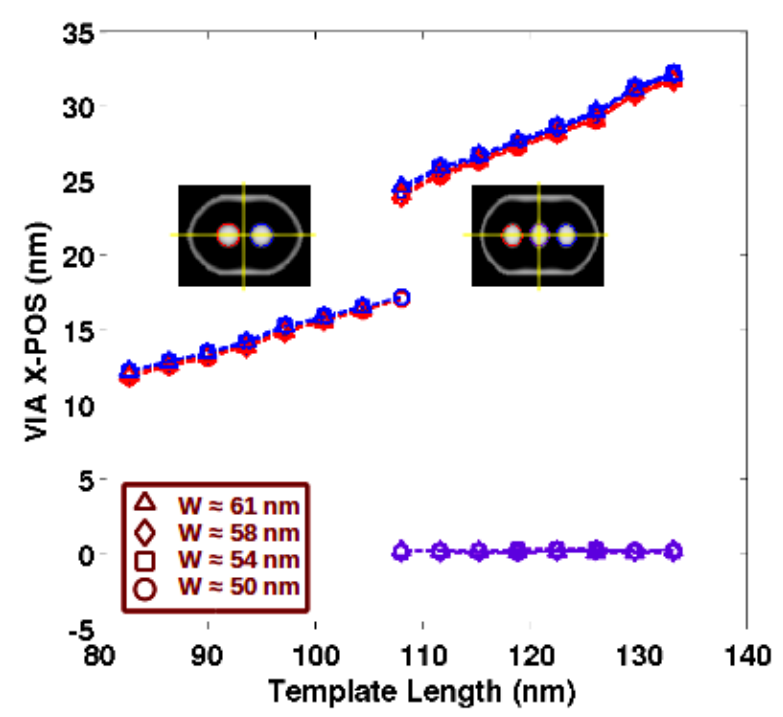

Figure 3: Same as Fig. 2 for the distance between the VIA centroid and the origin of the template (where the yellow lines cross).

find a V IA CD average growth rate of 0.14 and 0.1 for TwoCyl and ThreeCyl configurations, respectively.

\subsection{VIA Placement and Hole-to-hole distance}

The distance between the centroid of each VIA and the origin of the template (where yellow lines cross in Fig. 3) is plotted in Fig. 3 as a function of 


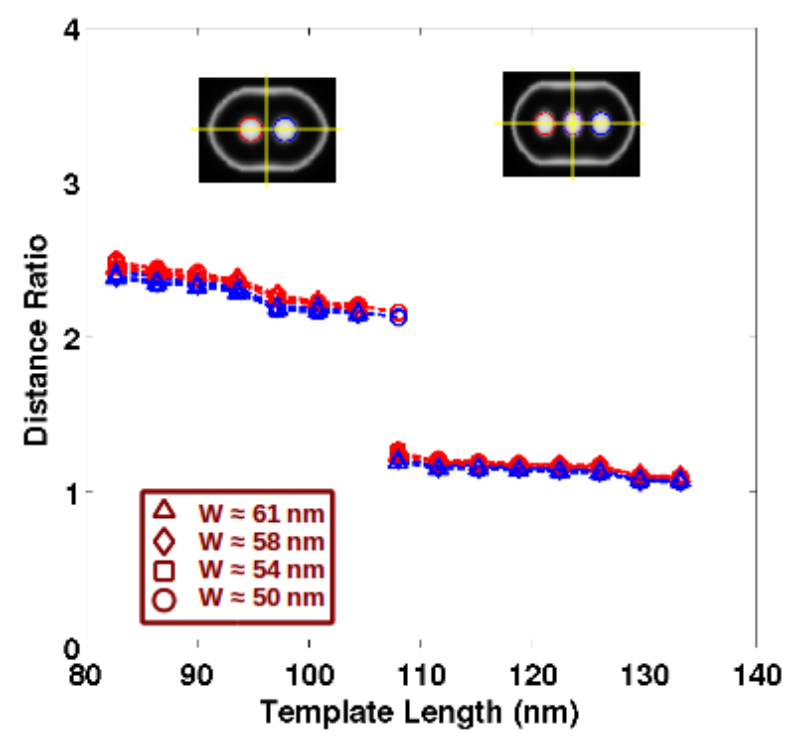

Figure 4: Same as Fig.2 for the ratio of the centroid-edge and centroid-origin distances.

template length for various template widths. Although not shown here, we found that the centroids of all VIA s are exactly on the horizontal yellow line located at half-width of the template. Hence, the reported distances in Fig. 3 are distances measured in the longitudinal direction of the template. As seen in Fig. 3, the VIA cylinders are symmetrically arranged inside the templates for both the TwoCyl and ThreeCyl configurations. The plots also show that the distance between the centroid of the end VIAS and the origin of the template are independent of width and grow linearly with length, indicating that end VIAS move away from the origin as the template length is increased. To further quantify the extent of the VIA displacement far from the origin, we plot in Fig. 4 the ratio of the distance between the centroid of an outer VIA and the closest edge of the template and distance between the centroid and the origin. Similar to the plots in Fig. 3, the ratio of centroid-edge and centroid-origin distances is identical for all template widths. However, as mentioned above, while the end VIAS move away from the origin when the template length is increased, Fig. 4 shows that the ratio of edge- to origin-centroid distances decreases slightly with the template length. In other words, the relative position of the end VIA centroid shifts towards the edges of the template with increasing length. As seen in Fig. 4, this phenomenon is more pronounced for TwoCyl configurations than

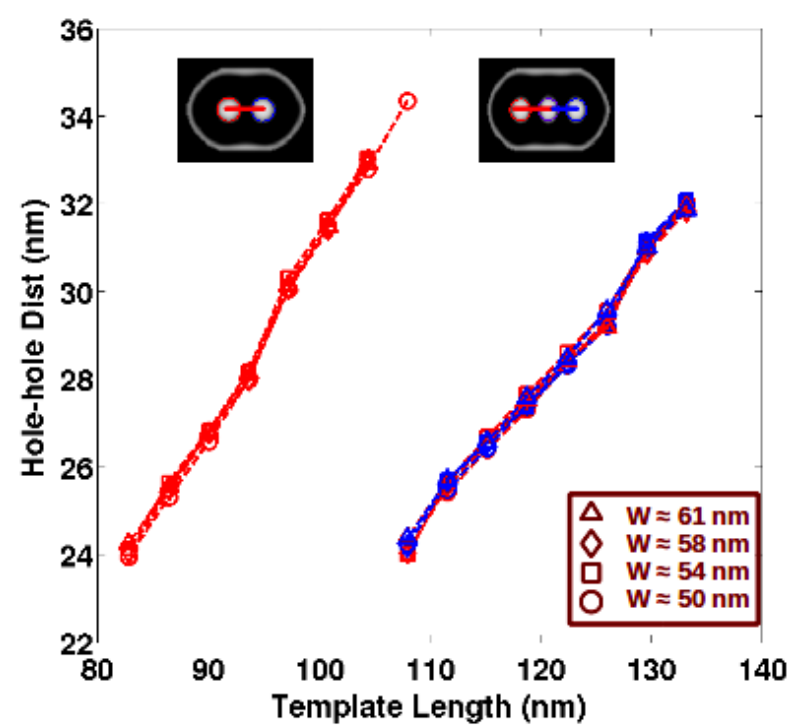

Figure 5: Same as Fig. 2 for the hole-to-hole distance.

ThreeCyl configurations where the relative shift or distance ratio is al most length-independent.

Using the centroid placement data in Fig. 3, we plot in Fig. 5 the hole-to-hole distance between nearest neighbor VIAs for TwoCyl and ThreeCyl configurations. Similar to the results above on V IA placement, the hole-to-hole distances are linearly increasing with the length of the template and independent of the width of the template. Our results also indicate that rows of contact holes can be produced in PS-PMMA with hole-to-hole distances between $24 \mathrm{~nm}$ and $34 \mathrm{~nm}$.

\section{Conclusion}

In conclusion, we have studied the self-assembly of PS-PMMA block copolymers by means of SCFT simulations in elongated templates and established commensurability windows for the formation of single rows of two and three cylindrical VIAs. Our results indicate that VIAS with increasing $C D$ form inside templates of increasing dimensions. The VIAs are symmetrically arranged inside the template and the resulting hole-to-hole distances range from $24 \mathrm{~nm}$ to $34 \mathrm{~nm}$ for template lengths between $80 \mathrm{~nm}$ and $140 \mathrm{~nm}$. We emphasize that the present work assumed perfect templates; future studies will examine VIA positioning within templates with line edge roughness. While encouraging for both $C D$ and placement aspects, our results nonetheless indicate low defect formation energies leading to 
defect densities well above the targets of the lithography community. The development of novel strategies, including alternative polymer architectures, to reduce defectivity is thus critical for the success of DSA in VIA lithography and contact multiplication.

\section{R eferences}

1. Herr, D. J. C., J. M ater. Res., 26 (2011) 122.

2. Segalman, R. A., M ater. Sci. Eng. R, 48 (2005) 191.

3. B ang, J., Jeong, U., Ryu, D. Y., Russell, T. P. and Hawker, C. J., Adv. Mater., 21 (2009) 4769.

4. Tiron, R., Chevalier, $X_{\text {., Couderc, C., }}$ Pradelles, J., Bustos, J., Pain, L., N avarro, C., M agnet, S., Fleury, G., and Hadziioannou, G., J. Vac. Sci. Technol. B, 29 (2011) 06F 206.

5. Takahashi, H., Laachi, N., Delaney, K. T., Hur, S.-M., Shykind, D., Weinheimer, C. and Fredrickson, G. H., Macromolecules, 45 (2012) 6253.

6. Laachi, N., Takahashi, H., Delaney, K. T., H ur, S.-M., Shykind, D., Weinheimer, C. and Fredrickson, G. H., Proc. SPIE, 8323 (2012) 83230K - 1.

7. Takahashi, H., L aachi, N ., H ur, S.-M ., Shykind, D., Weinheimer, C. and Fredrickson, G. H., Proc. SPIE, 8323 (2012) 83231N-1.
8. Tiron, R., Gharbi, A., A rgoud, M., Chevalier,

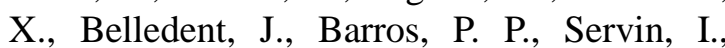
Navarro, C., Cunge, C., Barnola, S., Pain, L., A sai, M and Pieczulewski, C., Proc. SPIE, 8680 (2013) 868012.

9. Laachi, N., Delaney, K. T., Kim, B., Hur, S.-M ., B ristol, R., Shykind, D., Weinheimer, C. and Fredrickson, G. H., Proc. SPIE, $\mathbf{8 6 8 0}$ (2013), 868014-1.

10. Laachi, N., Delaney, K. T., K im B ., Hur, S.-M . Bristol R., Shykind, D., Weinheimer, C. and Fredrickson, G. H., J. Polym. Sci. Pol. Phys., in press (2014).

11. L aachi, N., Iwama T., Delaney, K. T., Kim B., Bristol R., Shykind, D., Weinheimer, C. and Fredrickson, G. H., Proc. SPIE, 9049 (2014) 904957.

12. Yi, H., Bao, X.-Y., Zhang, J., Tiberio, R., Conway, J., Chang, L.-W., Mitra, S., and Wong, H.-S. P., Proc. SPIE, 8323 (2012) 83230W-1.

13. Liu, C.-C., Pitera, J., Lafferty, N., Lai, K., Rettner, C., Tjio, M ., A rellano, N., and Cheng, J., P roc. SPIE, 8323 (2012) 83230X -1 (2012).

14. Fredrickson, G. H., [The equilibrium theory of inhomogeneous polymers], Oxford University Press, USA, (2006).

15. Bosse, A. W., Garcia-Cervera, C. J. and Fredrickson, G. H., Macromolecules, $\mathbf{4 0}$ (2007) 9570. 